\title{
Norois
}

Environnement, aménagement, société

190 | 2004/1

Varia

\section{Les ports de commerce bretons}

Des outils de proximité en mal de diversification

\section{Christophe Parreau}

\section{(2) OpenEdition}

Journals

Édition électronique

URL : https://journals.openedition.org/norois/70

DOI : $10.4000 /$ norois. 70

ISBN : 978-2-7535-1538-3

ISSN : $1760-8546$

\section{Éditeur}

Presses universitaires de Rennes

\section{Édition imprimée}

Date de publication : 1 janvier 2004

Pagination : 41-53

ISBN : 978-2-86847-939-6

ISSN : 0029-182X

\section{Référence électronique}

Christophe Parreau, «Les ports de commerce bretons », Norois [En ligne], 190 | 2004/1, mis en ligne le 01 septembre 2008, consulté le 14 janvier 2022. URL : http://journals.openedition.org/norois/70 ;

DOI : https://doi.org/10.4000/norois.70

Ce document a été généré automatiquement le 14 janvier 2022.

(c) Tous droits réservés 


\section{Les ports de commerce bretons}

Des outils de proximité en mal de diversification

\section{Christophe Parreau}

1 La Bretagne, baignée par l'océan Atlantique et la Manche, évoque spontanément l'image d'un territoire où la maritimisation semble s'imposer comme une caractéristique incontestable de l'économie. La puissance de la filière halieutique (environ $45 \%$ des apports français en volume et en valeur), le poids des activités touristiques (balnéaires et de plaisance) sur l'ensemble de son littoral ou encore celui de la construction navale qu'elle soit civile (à Lorient, à Saint-Malo, réparation navale à Brest) ou militaire (arsenaux de Lorient et de Brest) en témoignent.

2 En terme de commerce par voie de mer, cette maritimisation avérée doit en revanche être avancée avec beaucoup plus de prudence. Même si cette région est celle qui accueille le plus grand nombre de ports commerçant avec une certaine régularité à l'international (fig. 1), elle ne possède pas d'organisme majeur, aucun port ne s'affiche au-delà de 3 millions de tonnes de trafic, et sa participation à l'ensemble des flux portuaires métropolitains demeure faible (8,3 Mt soit $2,44 \%$ en 2000$)$. 
Figure 1 : Le trafic des ports de commerce bretons en 2000 (Direction du Transport Maritime des Ports et du Littoral [DTMPL])

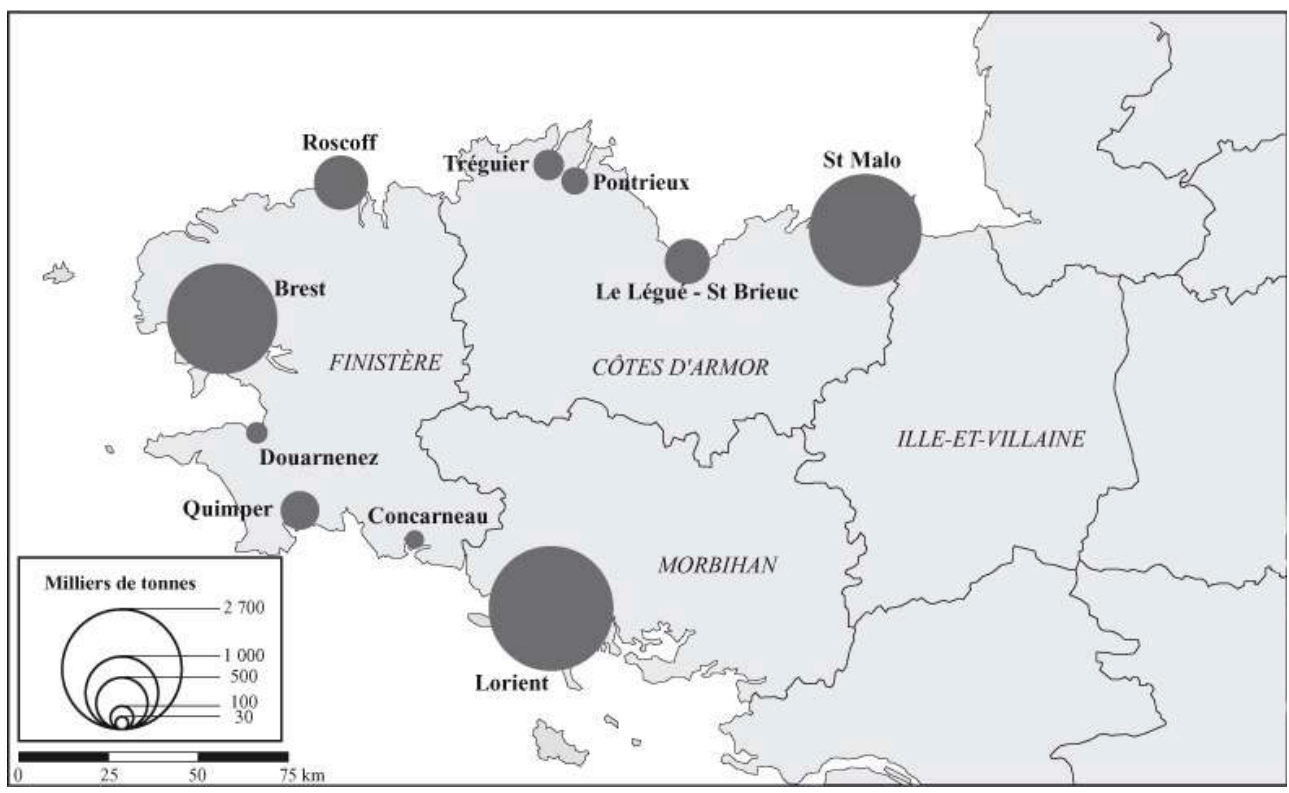

Géolittomer Nantes L.E.T.G. UMR 6554 du CNRS

Derrière cette indigence générale des courants maritimes, les ports bretons se singularisent toutefois par une spécialisation sectorielle en relation avec les aspirations de la première région agricole française qu'ils desservent. Cette orientation dominante d'origine continentale, qui se traduit parfois comme une quasi mono-activité, rejaillit ainsi sur la structure des flux maritimes dominée par les importations et caractérisée par une simplification des fonctions portuaires. En d'autres termes, l'activité des ports bretons, si elle s'inscrit en premier lieu dans un rapport de proximité lié à l'agroindustrie régionale, demeure cependant trop peu diversifiée, souffre d'une insuffisante structuration de ses horizons marins et traduit, en définitive, de façon encore plus accentuée la relative monovalence de la structure de l'économie régionale.

\section{Des ports de proximité avant tout animés par des fonctions de dépendance continentale}

\section{Une dépendance agro-industrielle}

Le secteur agro-alimentaire et ses activités connexes constituent des éléments fondamentaux dans l'animation de l'activité portuaire bretonne (tableau 1). Pour l'année 2000, l'ensemble des importations de vracs agro-alimentaires (aliments du bétail, graines oléagineuses, céréales, engrais et amendements marins) a représenté un trafic de plus de $3 \mathrm{Mt}$; soit près de $40 \%$ des courants maritimes bretons et $11 \%$ de l'ensemble des trafics agricoles ayant transité dans les ports français. Cette spécialisation agricole est ici très claire en comparaison avec le poids moyen réservé à cette filière dans l'ensemble des organismes maritimes français qui s'établit à $8,5 \%$ pour cette même année.

5 Les trois places portuaires bretonnes les plus importantes, Lorient, Brest et Saint-Malo, assurent près de $85 \%$ de ces courants à vocation agricole et agro-alimentaire soit une 
part identique au poids qu'elles détiennent dans l'ensemble des flux maritimes traités par les ports régionaux.

Tableau 1 : Part des vracs agro-alimentaires dans l'activité des trois principaux ports bretons en 2000 (en milliers de tonnes)

\begin{tabular}{|c|c|c|c|c|c|}
\hline Ports & Trafic total & $\begin{array}{c}\text { Vracs agro- } \\
\text { alimentaires }\end{array}$ & Hydrocarbures & $\begin{array}{c}\text { Part des vracs } \\
\text { agro-alim. }\end{array}$ & $\begin{array}{c}\text { Part des vracs } \\
\text { agro-alim. hors } \\
\text { hydrocarbures }\end{array}$ \\
\hline $\begin{array}{c}\text { Lorient } \\
\text { Brest }\end{array}$ & 2705 & 1172 & 1112 & $43,3 \%$ & $73,6 \%$ \\
\hline
\end{tabular}

\begin{tabular}{|c|c|c|c|c|c|c|}
\hline Port & Trafic Total & $\begin{array}{c}\text { Vracs } \\
\text { agro-alim. }\end{array}$ & Transmanche & Hydrocarbures & $\begin{array}{c}\text { Part des vracs } \\
\text { agro-alim. }\end{array}$ & $\begin{array}{c}\text { Part des vracs } \\
\text { agro-alim. hors } \\
\text { transmanche et hydrocarb. }\end{array}$ \\
\hline Saint-Malo & 2185 & 950 & 501 & 150 & $43,4 \%$ & $61,9 \%$ \\
\hline
\end{tabular}

(sources : DTMPL, 2000 ; Le Marin, avril 2001)

6 À Lorient et à Brest, ces flux sont constitués d'importations de produits destinés à l'alimentation animale qui se sont fortement développés à partir des années 1970. Ils répondent à la demande de la première région française d'élevage et à son industrie d'alimentation du bétail qui réalise plus de $40 \%$ de la production nationale. Les besoins de cette industrie, assurés en partie par des produits métropolitains (céréales, sons et issus, tourteaux de colza et de tournesol, pois et autres protéagineux...), le sont également par de forts tonnages de produits importés (tourteaux de soja, autres tourteaux, produits de substitution des céréales $\left.{ }^{1} . ..\right)$, essentiellement depuis le continent américain, qui transitent par les ports. Le degré d'incorporation de ces produits d'importation ${ }^{2}$ dans l'alimentation animale rythme ainsi d'année en année les résultats de ces organismes maritimes (fig. 2).

Figure 2 : Évolution des réceptions des matières premières destinées à l'alimentation animale dans les ports de Lorient et de Brest de 1985 à 2000
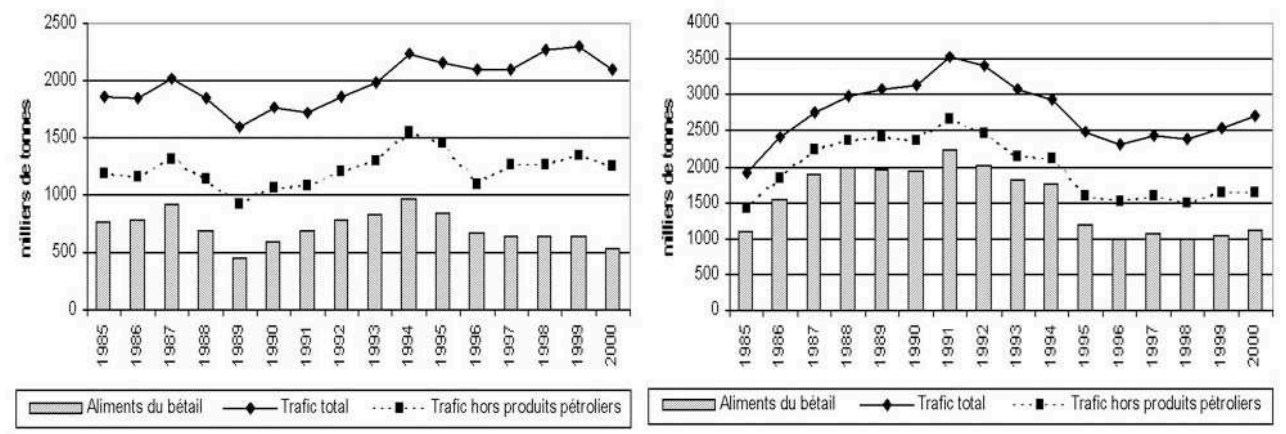

(sources : DTMPL, 1985-2000 et Le Marin, avril 2001)

7 L'utilisation plus massive de ces produits, à partir du milieu des années 1980, a globalement entraîné un accroissement des trafics. Cette tendance profita toutefois essentiellement à Lorient qui, en raison de sa position centrale sur le littoral de la Bretagne méridionale, bénéficie d'un hinterland potentiel réunissant 55 à $60 \%$ des capacités régionales de production d'aliments (fig. 3). Cette croissance soutenue (de 1 à plus 2,2 Mt entre 1985 et 1994) mais éphémère, comme nous le verrons plus loin, poussera encore davantage le port morbihannais dans une quasi mono-activité (plus de $60 \%$ de l'activité au milieu des années 1990, plus de $80 \%$ hors hydrocarbures) et 
d'autant plus qu'il ne pouvait faire l'économie d'installations spécialisées afin de s'adapter à la réception d'un éventail élargi de produits. À Brest, si la filière agroalimentaire tenait toujours un rôle notable, son évolution fut cependant plus contrastée eu égard à la relative étroitesse de son arrière-pays; étroitesse accentuée par ailleurs par un glissement progressif du centre de gravité de l'ensemble de la chaîne de production animale de l'ouest vers l'est de la région.

Figure 3 : Répartition des principales capacités de production d'aliments du bétail en Bretagne (en milliers de tonnes/an)

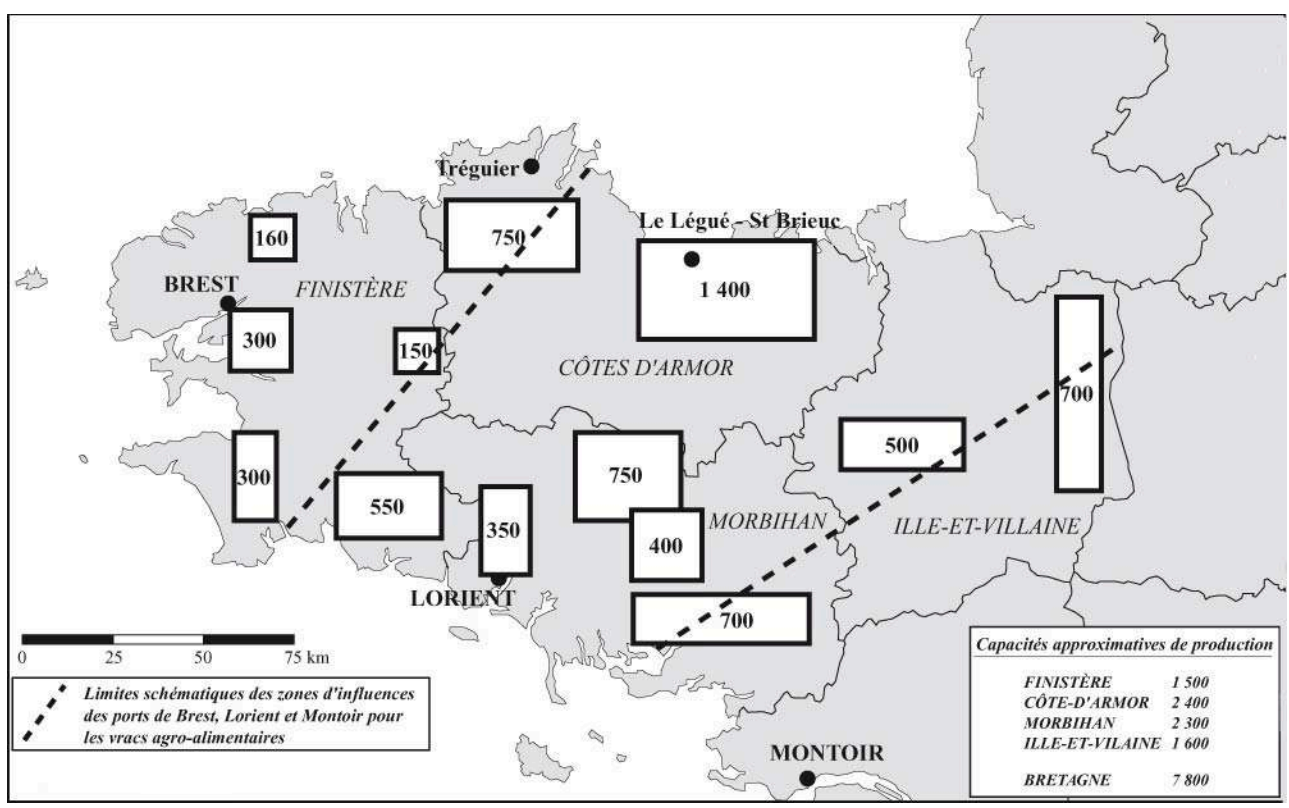

Sources diverses

Réalisation : Ch. Parreau

Géolittomer Nantes L.E.T.G. UMR 6554 du CNRS

8 Cette croissance, ayant permis l'établissement d'une certaine hiérarchie des places portuaires pour ces arrivages, cachait toutefois la fragilité d'une filière d'impulsion continentale extrêmement sensible à divers éléments conjoncturels (vache folle, poulets à la dioxine...) mais aussi et surtout structurels (Politique agricole commune, négociations dans le cadre de l'Organisation mondiale du commerce, risques environnementaux liés à la densité de la filière animale bretonne ${ }^{3}$ ). Ainsi, à la suite des négociations de l'Uruguay Round et de la réforme consécutive de la PAC au milieu des années 1990, la plus grande disponibilité des céréales communautaires sur le marché a profondément érodé les résultats de l'ensemble des ports bretons impliqués sur ces courants. À Brest, les importations de vracs agro-alimentaires sont ainsi passées d'1 Mt à $500000 \mathrm{t}$ entre 1994 et 2000, tandis que dans le même temps Lorient perdait un quart de son trafic global. Pour les petits ports de la Bretagne Nord intervenant sur des arrière-pays locaux, mais également comme soupapes du marché, la situation fut identique : Le Légué-Saint-Brieuc passait ainsi de 170000 tonnes d'aliments du bétail à 100000 tonnes entre 1995 et 2000, Tréguier enregistrant pour la même période une forte baisse, de 100000 à moins de 30000 tonnes.

9 Au-delà des ces évolutions mettant en évidence la dépendance des ports face à ces courants très fluctuants, il convient de souligner, et de façon plus générale, les effets somme toute réducteurs de ces vracs sur les économies portuaires (Guillaume, 1991). 
Même s'ils gonflent les résultats, ils demeurent peu générateurs de valeur ajoutée et d'emplois directs, ne sont pas ou peu révélateurs d'une quelconque agressivité commerciale puisque la nature des produits impose des acheminements terrestres les plus courts possibles et ne créent que peu de concurrence entre les places portuaires. Ils confinent ces dernières dans des fonctions de transit banales dont le champ d'action et le mode d'insertion géographique restent très simples. La structuration des arrièrepays des ports bretons impliqués dans ces transits de vracs agro-alimentaires est ainsi aujourd'hui déterminée essentiellement par la situation du port vis à vis des zones de productions. La situation était un peu différente jusqu'en 1994 puisque Lorient recevait un certain nombre de sous-produits (manioc, corn distiller, corn gluten...), en direct et en cargaisons complètes, dont il s'était fait une spécialité. Pour ces sous-produits son hinterland s'étendait alors à l'ensemble de la région Bretagne. Les réceptions s'étant aujourd'hui simplifiées (comme pendant les années 1970-1985 les importations sont aujourd'hui constituées essentiellement de tourteaux de soja), les arrière-pays le sont aussi ; ils sont redevenus éminemment captifs.

Comme le montre la figure 3 représentant les capacités des zones de production d'aliment du bétail et figurant schématiquement les zones d'influence des ports de Brest, Lorient et Montoir, l'arrière-pays brestois s'étend du Finistère au nord-ouest des Côtes-d'Armor tandis que celui de Lorient englobe le Finistère-Sud, les Côtes-d'Armor ainsi qu'une partie du Morbihan et de l'Ille-et-Vilaine; espaces sur lesquels (pour le sud-est du Morbihan et l'est de l'Ille-et-Vilaine) s'est imposée l'influence du terminal agro-alimentaire de Montoir la principale plate-forme pour cette filière. Les petits ports du Légué-Saint-Brieuc et de Tréguier, compte tenu de capacités nautiques limitées qui ne leurs permettent pas d'accueillir des navires supérieurs à 2 500-3 500 tpl, reçoivent pour leur part des cargaisons après transbordement, le plus souvent depuis les ports du Nord de l'Europe, et irriguent des hinterlands très locaux. Leurs arrière-pays naturels sont ainsi également alimentés, en partie, depuis Brest et Lorient qui reçoivent, quant à eux, des navires complets et en direct ce qui leurs permet, par économie d'échelle, de bénéficier de produits moins chers.

11 S'il existe, d'une manière générale, peu de concurrence entre les ports sur les principaux produits, et notamment sur les tourteaux de soja, il convient toutefois de souligner que le terminal de Montoir, qui traite aujourd'hui 2,2 Mt d'alimentation animale, accroît progressivement son influence sur certaines filières spécifiques. Les lourds investissements (traçabilité, traitement individualisés des lots, organisation et gestion du stockage sur le terminal...) consentis depuis plusieurs années par l'organisme autonome au terminal agro-alimentaire lui permettent en effet de prendre peu à peu quelques parts de marché à ses concurrents bretons et spécialement sur certains sous-produits (ceux-là même que Lorient traitait avant 1995) ou encore sur les filières nécessitant une traçabilité des produits. Cependant, la croissance du terminal de la Basse-Loire est avant tout à mettre en relation avec le développement de son hinterland vers le centre et le centre-ouest français. Le glissement progressif du centre de gravité de la filière animale bretonne de l'ouest vers l'est de la région bretonne, mais également en direction d'espaces limitrophes tels la Mayenne et la Loire-Atlantique, a également favorisé cette croissance.

12 À Saint-Malo, la fibre agricole s'exprime dans la réception d'engrais et de divers sousproduits entrant dans leur composition. Ces flux, réceptionnés par la Timac (filiale du groupe Roullier), représentent depuis plusieurs années autour de $60 \%$ (soit environ 
900000 tonnes) des trafics cargos réalisés dans les bassins de commerce du site malouin. La distribution des produits dans l'arrière-pays reste, quant à elle, essentiellement régionale; la Timac alimentant les multiples coopératives installées dans l'ensemble des départements bretons. Si ces courants sont moins fluctuants que ceux de vracs agro-alimentaires traités à Lorient ou à Brest, il n'en demeure pas moins que leurs caractéristiques restent identiques. Les installations occupent une part non négligeable de l'espace portuaire (espace fortement convoité dans un port comme celui de Saint-Malo inscrit dans la ville), la Timac profite d'une situation de quasi-monopole dans le port intérieur, les impacts en terme d'emplois portuaires sont faibles et ces trafics demeurent, enfin, suspendus à la santé de la filière qu'ils alimentent. Si les effets de l'essoufflement du modèle agro-industriel breton combinés à ceux des récentes crises sanitaires de la filière animale touchent encore peu le port malouin, qui bénéficie des économies d'échelle liées à la masse des trafics régulièrement manutentionnés, ils s'expriment en revanche clairement dans certains petits ports voisins. L'exemple de Pontrieux est caractéristique, puisqu'après avoir importé au milieu des années 1990 plus de 150000 tonnes d'engrais, trafic destiné dans sa totalité à l'usine Secma (filiale du groupe Roullier) installée sur les quais, il ne traite plus aujourd'hui que 90000 tonnes.

Qu'il s'agisse des réceptions d'aliments du bétail ou bien d'engrais, le rôle du port, ici utilisé comme simple outil, se réduit au transit de vracs destinés à des arrière-pays captifs ainsi qu'à quelques activités de stockage. Les variations de flux ne naissent en aucun cas de la concurrence interportuaire mais des mécanismes internes aux filières concernées. On imagine intuitivement le danger de cette situation dès lors que ces flux, souvent dominants, viennent à s'éroder, et particulièrement dans ces ports de taille moyenne où une part substantielle de l'espace portuaire leur est réservée. Lorient, par exemple, a été saturé à une époque où la demande était forte, mais aujourd'hui, alors que le trafic a fondu, les effets sont bien maigres. Les activités de quayage, de stockage ou bien encore de post-acheminement (dont le rôle se limite toutefois à de simples brouettages entre port et zones de production), qui s'étaient développées avec le trafic, se sont étiolées avec sa décroissance. Pire, cette hyper-spécialisation n'a jamais permis au port d'ouvrir quelques portes en direction d'une réelle diversification de son activité, garante d'une plus grande stabilité du site.

14 Ces fonctions de transit participent, il est vrai, à la compétitivité de l'ensemble de la filière agro-alimentaire régionale (un tiers des emplois industriels bretons) toutefois, ces relations restent la plupart du temps à sens unique puisque les installations de traitement des vracs n'ont aucune prise sur la récupération, et notamment à l'exportation, de courants plus nobles, de produits manufacturés issus ou non de cette branche d'activité.

\section{Les autres flux de vracs d'incitation locale et/ou régionale : un rôle important dans l'activité des ports bretons}

Dominée par des impulsions d'inspiration agricole, l'animation des ports bretons s'appuie également sur un certain nombre d'autres flux dont l'influence demeure également locale voire régionale. Conditionnés le plus souvent en vrac et portant, dans nombre de cas, sur des volumes substantiels, ces derniers utilisent à nouveau le port comme un simple espace de transit et le confinent dans des fonctions portuaires 
banales. Les services ou les fonctions que propose l'organisme portuaire ne sont pas ici réellement déterminants, mais c'est la seule proximité géographique qui permet d'y diriger quelques courants.

Au rang de ces trafics, le premier en volume concerne la redistribution d'hydrocarbures depuis les ports raffineurs français, mais également étrangers. À l'image de la plupart des ports moyens des littoraux atlantiques (Bayonne, Bordeaux et La Rochelle), Lorient et Brest traitent annuellement d'importants volumes destinés à alimenter leurs agglomérations en fuel domestique ainsi que les réseaux régionaux de distribution de carburants. Cette activité correspond ainsi, d'année en année, à environ $40 \%$ de l'activité globale de chacun de ces deux sites. À Saint-Malo, ces flux sont plus mesurés, s'affichant à moins de $10 \%$ des volumes totaux, tandis qu'à Concarneau et à Douarnenez (respectivement pour 2000, 30000 et 50000 tonnes alimentant les flottilles de pêche), ils représentent la quasi-totalité des trafics traités.

À côté de ces hydrocarbures, un éventail de vracs communs complète ces incitations de proximité (tableau 2). Nous pouvons évoquer brièvement les sables et agrégats marins destinés à l'industrie régionale du bâtiment (12\% de l'activité à Lorient, 23 \% à Roscoff, $70 \%$ à Quimper...), les ciments et clinkers (5\% des flux brestois), les exportations de kaolin depuis les petits ports des Côtes d'Armor ( $25 \%$ des courants traités au Légué, 7 \% à Roscoff...), les ferrailles... Bref, un ensemble de courants à faible valeur ajoutée qui ne génère, à nouveau, peu ou pas de concurrence entre les ports mais qui reste néanmoins significatif de l'activité de ces organismes maritimes bretons.

Tableau 2: Part des courants de vracs générés par des besoins locaux/régionaux dans l'animation globale des ports de commerce bretons en 2000 (y compris vracs agro-alimentaires) (en milliers de tonnes)

\begin{tabular}{|c|c|c|c|}
\hline Ports & $\begin{array}{c}\text { Part des vracs locaux/ } \\
\text { régionaux dans l'activité }\end{array}$ & Ports & $\begin{array}{c}\text { Part des vracs locaux/ } \\
\text { régionaux dans l'activité }\end{array}$ \\
\hline Lorient & $94 \%$ & Roscoff & $33 \%$ \\
Concarneau & $89 \%$ & Tréguier & $80 \%$ \\
Quimper & $100 \%$ & Pontrieuc & $100 \%$ \\
Douarnenez & $97 \%$ & Le Légué & $85 \%$ \\
Brest & $83 \%$ & Saint-Malo & $65 \%$ \\
\hline
\end{tabular}

(source : DTMPL, 2000 et Le Marin, avril 2001)

À la lecture de ce tableau qui cumule la part des vracs agro-alimentaires et celle des autres vracs répondant à des besoins locaux/régionaux dans les résultats globaux des ports de commerce bretons, force est de constater que celle réservée aux marchandises diverses est bien maigre à l'exception toutefois de Roscoff et de Saint-Malo qui opèrent sur le transmanche. À l'échelle de la Bretagne, ce sont en définitive plus de $80 \%$ des flux portuaires qui sont constitués par ces vracs ${ }^{4}$. Le constat est assez parlant quand on sait que la concurrence entre ports s'exprime essentiellement sur les marchandises diverses; ces trafics reposant le plus souvent sur l'existence de lignes régulières significatives d'une part, de la vitalité et d'une réelle structuration des horizons marins et d'autre part, de l'expression de stratégies portuaires portant sur la captation, voire la récupération, d'un certain nombre de produits manufacturés qui, avec la généralisation de la conteneurisation, ont tendance à rejoindre par route les ports mieux positionnés sur ces courants modernes (en l'occurrence ici essentiellement les sites du Havre et de la Basse Loire). 
19 On peut alors légitimement se demander si les ports bretons, et notamment les trois plus puissants, parviennent à s'extirper des spécialisations peu dynamisantes imposées par les contraintes de coût d'acheminement terrestre de ces vracs, s'ils sont capables de s'immiscer dans des filières plus enrichissantes et plus valorisantes.

\section{Des opportunités " naturelles » et commerciales ouvrant des possibilités de diversification de l'activité}

20 C'est aux marges de dominante, celle de la dépendance face aux vracs d'incitation agricole particulièrement, qu'il convient de déceler les tentatives, les opportunités et les potentiels d'évolution et de progrès de ces ports.

\section{Le transmanche en Bretagne Nord :une ouverture maritime sous contrôle des armements}

21 Les ports de Saint-Malo et de Roscoff opèrent sur le segment de la Manche Occidentale (avec Caen et Cherbourg); secteur qui assurait en 1998, pour l'ensemble des échanges transmanche français, $8,3 \%$ du trafic de passagers, $11,8 \%$ des véhicules de tourisme et $6,5 \%$ des véhicules commerciaux. Cette opportunité "naturelle " n'a cependant été exploitée que récemment, comparée à l'implication des ports de la Manche centrale et du Détroit, puisque c'est seulement à partir du milieu des années soixante-dix, sous l'influence de la Brittany Ferries, que l'offre s'est réellement développée et structurée, d'abord en Bretagne puis, dans les années 1980, en Basse-Normandie (Joan, 1998). Cette ouverture maritime en direction du sud du Royaume-Uni et de l'Irlande releva, encore une fois serait-on tenter de dire pour la Bretagne, d'initiatives venues de la terre et non de la mer.

22 À la fin des années 1950, le sous-équipement dramatique en matière de communications terrestres mais aussi maritimes, dont souffrait la région, était au centre des préoccupations des décideurs économiques et politiques régionaux (Le Rhyn, 1994). Leurs travaux, dans le cadre notamment du Comité d'Etudes et de Liaison des Intérêts Bretons ${ }^{5}$ (CELIB), se solderont par l'acceptation, le 9 octobre 1968, d'un plan d'équipement dit « Plan Breton ». À l'origine de ce plan, on trouvait les primeuristes du Léon, ceux-là même qui allaient quelques années plus tard devenir ces "paysansarmateur » en fondant la $\mathrm{BAI}^{6}$, qui créèrent, en 1963, une société d'études économiques sous l'impulsion de leur leader Alexis Gourvenec. Le «Schéma de structure du NordFinistère $^{7}$ » présenté par cette société d'études fut adopté par le département du Finistère puis repris par le CELIB dans une conception régionale sous l'étiquette de «Plan Breton ».

Activement relayé par la CCI de Morlaix et par la coopérative d'agriculteurs léonards, la SICA Saint-Pol-de-Léon, le projet d'édification d'un port roulier à Roscoff verra le jour en 1972. Jusqu'à cette date l'ancien port de Roscoff assurait l'exportation des productions agricoles locales (choux-fleurs, pomme de terre, oignons...) vers la GrandeBretagne mais la vétusté du site, combinée à des conditions nautiques limitées, avait conduit peu à peu les marchandises à transiter par les services de la compagnie transmanche Towsend-Thorensen implantée au Havre. Devant cette situation et grâce aux nouvelles infrastructures portuaires, la décision fut prise par la SICA Saint-Pol de 
créer sa propre compagnie maritime : avec l'achat d'un navire, le Kérisnel, la BAI voyait le jour en 1973.

À côté d'une première ligne réservée au fret entre Roscoff-Plymouth, la nouvelle compagnie maritime entendait bien développer un trafic de passagers compte tenu des croissances enregistrées dans les autres secteurs du littoral français de la Manche. L'arrière-pays de Roscoff étant limité pour le fret, mais également pour ces flux de passagers, ce nouveau service transmanche vit le jour, en 1975, entre Saint-Malo et Portsmouth (fin 1976, un service de fret fut ouvert à partir du port malouin).

À une croissance rapide des trafics passagers et fret dans les premières années d'exercice ${ }^{8}$ succédera cependant, dès le milieu des années 1980, une longue période d'adaptation et de mutation pour les deux ports bretons.

L'ouverture par la BAI de services transmanche à Cherbourg en 1985 puis à Caen en 1986 limitera en effet rapidement la progression des flux rouliers en Bretagne, et particulièrement pour le fret. À Saint-Malo, ces courants, tombés à 4000 camions en 1992, se sont cependant de nouveau développés suite à la mise en service en 1993 du nouveau terminal transmanche construit afin d'accueillir les navires de nouvelle génération ; ils s'établissent aujourd'hui aux alentours de 15000 unités de fret par an. À Roscoff, la concurrence des ports normands, combinée aux effets de la PAC sur les quotas laitiers poussant certains éleveurs britanniques vers une reconversion qui s'est faite, pour bon nombre d'entre eux, vers les primeurs réduisant ainsi les débouchés pour les productions bretonnes, a provoqué une érosion plus sensible du fret à partir du début des années 1990. Actuellement, Roscoff enregistre moins de 5000 unités de fret par an. En comparaison, les courants de fret traités par la BAI s'élèvent aujourd'hui à 75000 unités à Caen et à 35000 à Cherbourg.

Les terminaux ferry des deux ports bretons se sont ainsi progressivement transformés, sous la houlette des compagnies maritimes ${ }^{9}$, en terminaux essentiellement animés par une activité touristique saisonnière. Ces trafics s'élèvent aujourd'hui à 602000 passagers à Roscoff (460 000 vers l'Angleterre et 142000 vers l'Irlande) et à 593000 à Saint-Malo. Peu touchée par les effets de l'ouverture du tunnel sous la Manche, le segment Manche Occidentale étant situé en dehors de sa concurrence directe, l'activité qu'entretiennent Roscoff et Saint-Malo avec leurs pendants britanniques et irlandais demeure aujourd'hui relativement stable et ce, malgré la récente disparition du duty

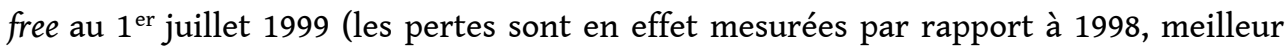
exercice pour les deux sites : 40000 passagers en moins à Roscoff et 5000 en moins à Saint-Malo).

Si cette ouverture maritime profite aujourd'hui à l'économie touristique régionale, puisqu'il est avéré que la grande majorité des passagers britanniques et irlandais (qui correspondent à environ $75 \%$ des passagers transitant par les ports transmanche du Nord de la Bretagne) séjournent pour $90 \%$ d'entre-eux dans l'Ouest français, les effets strictement portuaires sont cependant à nuancer. D'une part, nous l'avons vu, l'offre de fret reste limitée ${ }^{10}$ et la finalisation de l'autoroute des estuaires devrait encore davantage orienter les marchandises en provenance de l'Ouest français, voire de la péninsule ibérique, vers les ports bas-normands. Par ailleurs, quelle valeur donner aux tonnages générés par le transmanche, 500000 tonnes de trafic à Saint-Malo et près de 300000 tonnes à Roscoff, quand on sait qu'ils correspondent, pour une large part, au poids des véhicules de tourisme? 
Enfin, il convient de souligner que l'activité transmanche dépend avant tout des stratégies des compagnies maritimes qui la contrôlent. Le port se trouve alors dans une nouvelle situation de dépendance, puisque s'il reçoit une part substantielle de ses ressources de cette activité au travers des droits de ports acquittés à chaque rotation de navire, il en réinvestit une part non négligeable pour s'adapter sans cesse aux évolutions technologiques caractéristiques de ce marché. Ce sont en fin de compte ces évolutions qui incarnent la véritable concurrence qui existe sur cette activité : celle de la capacité des armements à renouveler leur flotte. Au centre de cette concurrence que se livrent les opérateurs maritimes, le port se transforme alors le plus souvent en simple outil qui doit répondre aux exigences des premiers sous peine de voir ses trafics s'étioler mais aussi, en simple port couloir traversé par des flux qui n'ont pas nécessairement d'impacts économiques locaux significatifs.

\section{Brest : l'exception bretonne dans l'élargissement et la structuration des horizons marins}

30 Nous l'avons précédemment souligné à plusieurs reprises, la filière agro-alimentaire apparaît plus stérilisante que dynamisante pour les ports qu'elle emprunte puisque sa présence se résume au seul transit d'importants volumes de vracs secs, souvent fluctuants, mais dévoreurs d'espace. En retour, les produits alimentaires transformés semblent s'écarter des ports moins éminents au profit de la route, quand il s'agit du marché européen, ou bien d'organismes portuaires plus puissants, quand il s'agit d'exportations vers des pays tiers. Dans la quasi-totalité des ports bretons, ces tonnages de produits alimentaires sont ainsi relégués à quelques cargaisons annuelles représentant quelques centaines voire quelques milliers de tonnes. Un port, celui de Brest, déroge toutefois à cette règle. Il s'érige même depuis plusieurs années au rang de premier port européen pour les exportations de viandes congelées. Cette activité ancienne, établie sur l'existence de plusieurs lignes régulières, a par ailleurs été à l'origine d'un développement récent de la diversification des services maritimes réguliers dont bénéficie aujourd'hui le port finistérien.

31 Cette situation trouve à nouveau son origine à terre. Le groupe Doux, puissant groupe volailler implanté à une quarantaine de kilomètres de Brest, assure en effet lui-même, depuis la fin des années 1960, ses exportations de volailles congelées au départ du port de Brest avec sa propre filière maritime, la West Reefer Line, fixant ainsi de forts courants réguliers. Il fut rejoint sur les quais brestois quelques années plus tard par deux autres producteurs volaillers, Tilly (filiale du groupe Bourgoin) et Sabco (filiale d'Unicopa) qui chargent, pour leur part, leurs productions sur les navires de l'armement danois Lauritzen. À partir du début des années 1980, Brest bénéficiait ainsi de deux lignes régulières (Brest-Moyen Orient assurée par la West Reefer Line et Danemark (via Brest)-Moyen-Orient assurée par Lauritzen) pour un trafic de viandes congelées établi à plus de 200000 tonnes. Toutefois, quelques années plus tard, la concurrence des productions nord-européennes, un certain tassement des marchés traditionnels moyen-orientaux (quelques pays clients ayant investi dans l'élevage volailler afin de se suffire à eux-mêmes) mais aussi, et surtout, l'insuffisante diversification à la fois technique et géographique de l'offre maritime au départ de Brest provoqueront une érosion sensible des flux. Avec l'avènement du conteneur, une part significative des trafics de volailles, et plus généralement de viande et de produits 
alimentaires, utilisait en effet plutôt les quais havrais, nantais, voire anversois, pour rejoindre leurs destinations finales.

Les ambitions de la CCI de Brest, consciente de la nécessité de projeter véritablement le port dans l'ère du multimodalisme, afin de bénéficier d'une partie du potentiel de l'arrière-pays agro-industriel breton, combinées à l'esprit pionnier du groupe Doux qui amorcera, en 1996, un premier trafic régulier de conteneurs, permettront de redynamiser, et même de diversifier, à la fois les produits exportés et les destinations touchées.

Les premières actions de l'organisme concessionnaire en faveur de ces trafics s'exprimèrent ainsi, au début des années 1990, par un renforcement des superstructures destinées au stockage des viandes congelées, ainsi que par une politique de prospection vers d'autres produits alimentaires bretons susceptibles d'utiliser les lignes régulières existantes. De 1992 à 1995, profitant d'une conjoncture favorable mais aussi des premiers effets de ces démarches commerciales, et particulièrement sur des volumes de viandes congelées bretonnes autres que les volailles, les trafics reprendront une courbe à la hausse. Dès 1996, la diminution des aides à l'exportation décidée à Bruxelles stoppera toutefois cette embellie.

Cependant, cette année 1996 fut marquée par les prémices d'une future relance des exportations. La filière maritime du groupe Doux qui travaillait jusqu'alors avec quatre navires polythermes sur des trafics palettisés en direction du Moyen-Orient, mit en service deux navires polyvalents permettant de charger une cinquantaine de conteneurs en pontée. Dès leur première année d'exercice, un trafic mensuel d'environ 100 Evp fut créé. En outre, cette offre conteneurisée offrait alors à certains clients, qui utilisaient précédemment les services du Havre faute d'un service adapté en Bretagne, de nouvelles possibilités pour leurs expéditions de produits laitiers, de viande, de plants de pomme de terre... Parallèlement à ces premiers pas dans la conteneurisation, la CCI finalisait son projet de plate-forme multimodale entre 1996 et 1997 (terre-plein, poste roulier, outillage destiné à la manutention des conteneurs...). Les résultats ne se feront pas attendre : à la fin de l'année 1997, une nouvelle ligne régulière reefer, opérée par l'armement Northern Shipping, fut ouverte en direction de la Russie et, en décembre 1997 et 1999, Maersk puis la CMA-CGM intégraient Brest comme escale sur trois lignes feeder connectant ainsi le port finistérien et son hinterland au reste du monde.

$35 \mathrm{Au}$ total, Brest dispose aujourd'hui de quatre lignes régulières reefer et d'une ligne feeder (les services bimensuels de la CMA-CGM ayant été arrêtés au printemps 2000) qui lui ont permis, en 1999, de traiter un trafic de 280000 tonnes de viandes congelées et de plusieurs milliers de tonnes d'autres produits alimentaires. D'autre part, à l'avant-pays traditionnel concentré sur le Moyen-Orient se sont ajoutés de nouveaux horizons marins comme la Russie, Cuba ou encore l'Afrique du Sud avec laquelle West Reefer Line travaille de plus en plus dans le but de charger du fret à la remontée de ses navires. Enfin, le développement du feedering permet aujourd'hui de positionner le port comme détenteur d'un gisement de fret identifié et maîtrisé ; il devient plus acteur que simple outil. Le trafic de conteneurs, inexistant au milieu des années 1990, a d'ailleurs rapidement progressé de 8000 Evp en 1998, à 11000 en 1999 et à près de 15000 aujourd'hui.

36 Même si elle n'est pas entièrement responsable de la réussite brestoise dans le multimodalisme, on peu toutefois constater que la niche que constituent les 
exportations de volailles y a activement participé. Pionnière sur ces nouveaux conditionnements à Brest, elle offre l'exemple très significatif de l'effet d'entraînement d'une activité à forte valeur ajoutée et à fort potentiel, structurée de longue date sur le plan maritime, ayant entraîné une sorte d'effet boule de neige en attirant de nouveaux trafics et de nouveaux opérateurs maritimes.

\section{Le cabotage de lignes régulières intra-communautaire : une réelle opportunité de diversification et de développement ?}

Compte tenu d'une part de la situation géographique de la Bretagne encadrée au sud par la Basse-Loire et au nord par la Basse-Seine, principale plate-forme française pour les trafics de conteneurs transcontinentaux, et d'autre part, des politiques logistiques actuelles des grands armements conteneurisés qui cherchent à intégrer le maillon terrestre dans une prestation de bout en bout ${ }^{11}$, les opportunités de développement pour les ports secondaires bretons sur les marchandises diverses semblent ainsi tenir davantage dans l'opportunité $d u$ développement de lignes régulières intraeuropéennes. L'essor de ce cabotage de lignes régulières s'inscrit en effet dans les orientations européennes actuelles en faveur d'une nécessaire redistribution modale des échanges intra-communautaires. Cependant, malgré cette volonté ${ }^{12}$ et les attentions dont bénéficie le mode maritime depuis plusieurs années, les résultats en terme de création de lignes, demeurent encore à ce jour limités.

Cette situation s'explique tout d'abord, et d'une façon générale, par la concurrence du mode routier qui reste hégémonique en Europe pour le transport de marchandises diverses. Ce mode profite notamment de sa nature qui lui confère une grande adaptabilité et une grande flexibilité, critères essentiels au regard des chargeurs qui, à l'inverse, perçoivent souvent le maritime comme un mode d'acheminement lent, complexe dans ses opérations, parfois obscur dans son fonctionnement (rôle réel des intervenants) et souvent opaque quant à ses coûts. Par ailleurs, il n'est pas inutile de rappeler que l'hégémonie du mode routier s'est également appuyée sur une situation privilégiée lui permettant de ne pas supporter l'ensemble de ses coûts de fonctionnement (coûts externes relatifs aux infrastructures routières, coûts environnementaux...).

Les difficultés d'essor des liaisons maritimes régulières trouvent aussi leur source dans un certain nombre de contraintes financières liées aux moyens et au temps nécessaires à l'établissement d'un courant de fret et à sa pérennisation, contraintes très souvent à l'origine de la fermeture de liaisons nouvellement créées. En dehors du cas brestois, d'autres tentatives de diversification de l'activité ont vu le jour au cours des années 1990 en Bretagne. Ce fut notamment le cas à Lorient qui s'équipa d'une passerelle ro/ro dès 1991. Les lignes roulières Lorient-Gijòn (montée en 1991) puis Lorient-Casablanca (1996), alternatives au " tout routier » entre les marchés de la Péninsule ibérique et du Maroc et ceux du Nord de la France et du Royaume-Uni (en limitant, pour ce dernier, le parcours terrestre à la liaison entre Lorient et les services ferry de Saint-Malo ou de Roscoff), se solderont cependant par deux échecs après quelques mois d'exploitation. On imputa ces revers à la frilosité des chargeurs bretons, l'explication étant sans doute aussi, et surtout, à trouver dans l'étroitesse des structures financières encadrant ces projets, comme dans nombre de projets locaux initiés au sein de ports moins éminents, 
qui se sont avérées incapables de supporter une défaillance, même temporaire, des taux de remplissage nécessaires au seuil de rentabilité des lignes.

Les programmes européens (Programme PACT puis Marco Polo depuis 2001) de soutien aux nouveaux services maritimes pendant une période de mise en place semblent aujourd'hui proposer un cadre plus favorable à leur essor. Toutefois, reste à savoir si d'éventuels projets de lignes intégrant les ports bretons pourront bénéficier à court terme de ces outils dans la mesure où les principales préoccupations européennes sont aujourd'hui légitimement focalisées sur des points de congestion majeurs tels les franchissements alpins ou pyrénéens. Par ailleurs, afin qu'elle porte ses fruits, il convient aussi de souligner que cette opportunité du cabotage intra-européen doit participer d'un vaste projet d'intérêt général dans lequel il faudra sans doute, outre ces efforts de soutien au démarrage des lignes, que les États agissent afin d'équilibrer effectivement la concurrence entre les modes en freinant notamment, par diverses mesures, le développement de la route.

41 Enfin, comme il a été souligné précédemment, la forte spécialisation de la plupart des ports de commerce bretons dans les vracs agro-alimentaires explique également en partie l'absence ou tout au moins la difficulté de fixer ces lignes régulières. En d'autres termes, ces ports ne reçoivent que les courants pour lesquels ils ont été conçus. Malgré le développement récent d'outils destinés à la manutention de marchandises unitisées, les communautés portuaires sont encore peu impliquées dans ces filières et les démarches commerciales, celles qui créent puis participent à fidéliser les trafics, restent donc à être développées dans nombre de cas.

\section{Conclusion}

En Bretagne, Brest est aujourd'hui le seul port de commerce à avoir véritablement posé les premières pierres dans la voie d'une diversification de ses activités. Il est le seul à disposer de lignes régulières, à profiter d'escales feeder ; en d'autres termes, à être relié au reste du monde. Pour les autres, c'est davantage la permanence qui domine, celle d'un repli sur des flux peu nobles de dépendance continentale destinés à des arrièrepays de proximité quasi exclusifs. Sur le littoral septentrional, le transmanche extrait en partie Roscoff et Saint-Malo de ces courants de proximité, toutefois cette activité manifeste aujourd'hui certaines limites eu égard à la position « excentrée » des deux ports rouliers bretons sur ce segment.

43 Ainsi, si les ports régionaux participent à la maritimisation de l'économie bretonne cela s'exprime davantage en termes quantitatifs que qualitatifs. L'exemple breton illustre en effet combien il est difficile pour des ports de taille moyenne, dont les difficultés d'insertion sur des filières à forte valeur ajoutée sont manifestes, d'affirmer de véritables stratégies tendant vers une plus grande valorisation de leurs fonctions.

La vive promotion actuelle à l'échelle européenne du short sea shipping face aux nuisances du mode routier, même si elle nécessite encore une réflexion des États sur les moyens politiques à mettre en œuvre afin de réaliser effectivement un rééquilibrage entre les modes d'acheminement, constitue sans doute une opportunité pour ces ports secondaires. Cependant, et afin de saisir cette opportunité, il faut dès à présent que les décideurs politiques et économiques bretons tentent de redonner une dynamique 
maritime à leur région plus tournée vers la terre que vers la mer depuis déjà plusieurs décennies.

\section{BIBLIOGRAPHIE}

COPETRANS, 2001. - « Étude sur les flux routiers transférables vers des schémas de transport maritime dans les régions atlantiques ", 124 p., dans Pour le renouveau du cabotage sur la façade Atlantique, CESR de l'Atlantique, Nantes.

Guillaume (J.), 1991. - « Les trafics agricoles dans les fonctions portuaires de la façade Atlantique », Revue Géographique du Centre-Ouest, n³, p. 67-73.

JoAN (J.-M.), 1998. - Les liaisons transmanche, Paris, Hermes, Coll. « transports et sécurité », 209 p.

LE RHUN (P.-Y.), 1994. - Géographie et aménagement de la Bretagne, Morlaix, Skol Vreizh, 239 p.

PARREAU (Ch.), 2001. - Les ports de commerce moyens du versant Manche-Atlantique français : étude géographique, Thèse de doctorat, Université de Nantes, 2 vol., 487 p.

\section{sources statistiques}

Direction du Transport Maritime des Ports et du Littoral (DTMPL), 1985-2000, Résultats de l'exploitation des ports maritimes (rapports statistiques annuels), ministère de l'Équipement des Transports et du Logement, Paris.

Le Marin, 2001, Ports de commerce. Bilan 2000, hors série, Rennes, Infomer.

\section{NOTES}

1. Produits de substitution des céréales (PSC) : manioc, corn-gluten feed, corn distiller (déchets de l'amidonnerie du maïs)...

2. Les céréales métropolitaines, entrant généralement pour $50 \%$ dans la composition des aliments composés jusqu'en 1985, tomberont à environ $30 \%$ en 1990 au profit de nouveaux produits importés. En Bretagne, ce phénomène sera encore plus accentué avec des taux d'incorporation de céréales métropolitaines, et notamment dans l'aliment porcin, tombant à 15 ou $20 \%$; les PSC représentant, quant à eux, jusqu'à $50 \%$ des produits utilisés.

3. À titre d'illustration, la densité de production d'aliments du bétail au $\mathrm{km} 2$ (indicateur indirectement révélateur de la concentration des structures de production animale) est de $35 \mathrm{t} /$ km2 à l'échelle de l'ensemble du territoire français tandis qu'elle s'élève à $275 \mathrm{t} / \mathrm{km} 2$ pour la Bretagne (soit 8 fois plus), à $93 \mathrm{t} / \mathrm{km} 2$ pour les Pays-de-Loire seconde région française et à $64 \mathrm{t} /$ $\mathrm{km} 2$ pour le Nord-Pas-de-Calais qui occupe le troisième rang.

4. La part des marchandises conditionnées en vrac représente, pour 2000, $74 \%$ de l'ensemble des flux portuaires métropolitains.

5. Le CELIB obtint en 1956 le premier plan de développement régional lancé en France. Ce plan prévoyait notamment un port pétrolier et une raffinerie à Brest, le Plan Routier Breton (un 
ceinturage de la péninsule par une voie express à deux fois deux voies reliant les villes littorales entre elles et cet ensemble à Rennes ainsi qu'un aménagement progressif des liaisons intérieures) ainsi que la construction d'un port en eau profonde à Roscoff.

6. Bretagne-Angleterre-Irlande, raison sociale de la société d'exploitation depuis ses débuts; Brittany Ferries désignant, depuis 1974, la marque commerciale du groupe.

7. Il comportait deux axes: industrialisation du Finistère pour offrir des emplois aux petits paysans dont le départ devait permettre d'agrandir les exploitations restantes et désenclavement de la Bretagne pour l'intégrer dans le marché européen (liaisons routières vers le Bénélux et l'Espagne et liaisons maritimes vers la Grande-Bretagne).

8. En 1980, 12000 camions, 250000 passagers vers l'Angleterre et 50000 vers l'Irlande transitèrent par Roscoff tandis que Saint-Malo enregistrait, pour cette même année, 350000 passagers et 12700 véhicules commerciaux vers l'Angleterre.

9. Irish Ferries s'est implanté à Roscoff en 1995 tandis que Condor Ferries, spécialisé jusque-là dans les liaisons avec les îles Anglo-normandes, a ouvert en 1998 à Saint-Malo des liaisons saisonnières en direction de Poole et de Weymouth.

10. Pour ne s'en tenir qu'aux exportations issues de la région Bretagne, le bureau d'études COPETRANS estime que près de $60 \%$ des courants dirigés vers le Royaume-Uni en 1999, flux essentiellement constitués de produits transformés issus de la filière agro-alimentaire, utilisent des ports extra-régionaux (Copetrans, 2001).

11. Cette volonté actuelle d'intégration de l'ensemble de la chaîne de transport, et particulièrement à terre, se traduit par un maillage du territoire par implantation de multiples dépôts de conteneurs. Ces dépôts permettent aux armements d'optimiser la gestion de leur parc de boîtes et, pour ceux implantés au Havre par exemple, de recruter leur fret par route aux portes des ports bretons (ce qui réduit les possibilités d'implantations de nouveaux services feeder) et même de la Basse-Loire.

12. Voir notamment les références suivantes: Le développement du transport maritime à courte distance en Europe: une alternative dynamique dans une chaîne de transport durable, COM (1999) 317 final, Bruxelles, juin 1999; La politique européenne des transports à l'horizon de 2010 : l'heure des choix, Livre Blanc, Bruxelles, 2001.

\section{RÉSUMÉS}

L'animation des ports de commerce bretons se caractérise par une forte spécialisation dans les importations de vracs agro-alimentaires en relation avec l'agro-industrie régionale. Les ports répercutent ainsi de façon accentuée, à travers cette spécialisation frisant parfois la quasi monoactivité, la relative monovalence de la structure de l'économie régionale. Au-delà de ces impulsions majeures, nombre d'entre eux peinent à diversifier leur activité et leurs horizons marins en fixant notamment des courants réguliers de marchandises diverses à forte valeur ajoutée ; celles-ci empruntant le plus souvent des ports extra-régionaux ou la route. Aujourd'hui, seul Brest, implanté de longue date sur les exportations de viandes congelées, bénéficie de l'expérience acquise dans l'organisation de cette niche de trafic pour poser véritablement les premières pierres en direction du multimodalisme. En Bretagne septentrionale, l'opportunité «naturelle» offerte par le transmanche participe également à la diversification des activités portuaires même si elle se traduit par une forte dépendance des ports face aux opérateurs maritimes. 
The activity of Breton commercial ports is characterized by a strong specialization in the imports of agro-alimentary bulks linked to regional agricultural processing industry. The ports thus reflect in an accentuated way the relative monovalence of the structure of regional economy. Beyond these major impulses, numbers of them face difficulties diversifying their activities and their marine foreland by fixing regular shipping lines of general cargoes at strong added value. Today, only Brest, long-established on frozen meat exports, profits from the experience gained in the organization of this specific trade towards multimodalism. In septentrional Brittany, the natural opportunity offered by the cross-Channel trading also takes part in the diversification of the harbour activities even if it results in a dependence of the ports on maritime operators.

INDEX

Index géographique : France, Bretagne

Mots-clés : arrière-pays, port, avant-pays, transmanche

Keywords : cross-Channel trading, hinterland, port, maritime transport

\section{AUTEUR}

\section{CHRISTOPHE PARREAU}

Docteur en Géographie, Université de Nantes, LETG-UMR 6554 - CNRS, chris.parreau@infonie.fr 